\title{
Neurologie und Psychiatrie: Brauchen wir das andere Fachgebiet?
}

n den letzten 20 Jahren haben sich die Nachbarfächer so weit auseinander entwickelt, dass vielerorts sogar die Forderung laut wird, man könne auf das „Pflichtjahr“ verzichten. Dabei waren sich die beiden Fächer meines Erachtens noch nie so nah wie jetzt und vielleicht werden die nächsten Generationen die Einteilung in zwei Fächer sogar belächeln. Schon lange hat sich die biologische Psychiatrie fest etabliert und die Ergebnisse der Genetik, Bildgebung und operativen Therapie in der Psychiatrie zeigen höchst interessante Ergebnisse. Viele neurologische Erkrankungen gehen mit psychiatrischen Symptomen einher, stellenweise treten diese sogar in den Vordergrund. Am deutlichsten wird die Nähe in der Pharmakotherapie. Wir sprechen hier über die gleichen Transmitter und Rezeptoren und wohlgemerkt auch über die gleiche Anatomie und Pathophysiologie. Im niedergelassenen Bereich überwiegen sogar die Nervenärzte und Ärzte mit beiden Zusatzbezeichnungen. Weshalb also diese Dichotomie?

Sicherlich liegt dies auch an den Ressentiments der Ausbilder, die Bedenken gegen Mitarbeiter der anderen Fachdisziplin haben. Aber auch an der Ausbildungsordnung, die nicht gewährleistet, dass man wesentliche Aspekte des anderen Fachs vermittelt bekommt. Zuletzt vielleicht auch, weil das eigene Fach schon viel zu umfangreich ist.

Lassen Sie uns von der Vielfältigkeit des Fachs profitieren und unsere Arbeit von den Nachbardisziplinen befruchten. In ein Neurozentrum gehören neben Neurologie und Psychiatrie auch Neurochirurgie, Neuroradiologie, Neuropathologie und Psychologie. Eine Zeitschrift, die psychoneuro heißt, sollte dieser Forderung ebenfalls Rechnung tragen, weshalb wir mit dem vorliegenden Heft etwas das Grenzgebiet der beiden Fächer Neurologie und Psychiatrie beleuchten. In dem Beitrag von PD Dr. Müller, der nach seiner psychiatrischen, neurologischen und neuroradiologischen Ausbildung in der Forensik arbeitet, geht es um die Neurobiologie der Forensik, die er mit zwei äußerst interessanten Kasuistiken verdeutlicht. Der Beitrag von Dr. Kessler und Prof. Falkai be- leuchtet die Lewy-Körperchen-Demenz, die einerseits ein ideales Feld für die Zusammenarbeit von Psychiatrie und Neurologie ist, aktuell aber auch noch für viel Diskussion zwischen den beiden Fächern sorgt. Die Autoren sind prädestiniert, den Beitrag aus interdisziplinärer Sicht zu schreiben, da gerade am Homburger Neurozentrum die verschiedenen Fachdisziplinen auf diesem Forschungsgebiet intensiv zusammen arbeiten. Den dritten Beitrag verfasste Prof. Steinhoff vom Epilepsiezentrum Kork. Gerade in der Abgrenzung von psychogenen und organischen Anfällen sind interdisziplinäre Kenntnisse unabdingbar. Aber auch in der Therapie erschöpfen sich die Maßnahmen sicherlich nicht mit der Gabe von Medikamenten.

Die Frage, ob sich die beiden Fächer zukünftig noch weiter trennen sollten, muss man kritisch diskutieren. Dabei muss auch geklärt werden, ob das psychiatrische/neurologische Jahr in der Ausbildung zum Neurologen/Psychiater noch sinnvoll ist. Meines Erachtens sollte man darauf nicht verzichten. Zum Vorteil der Ausbildung, zum Vorteil unserer Patienten.

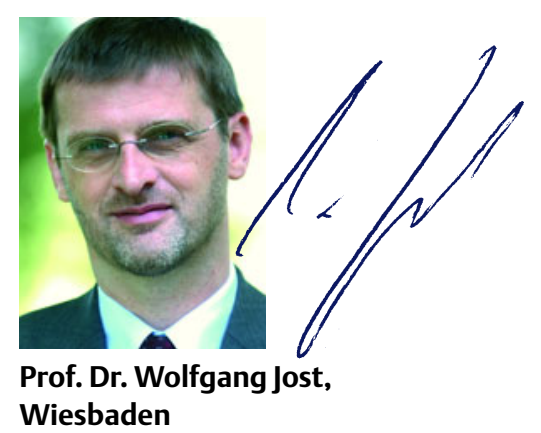

